SIMULATIONS OF TURBULENCE

INDUCED ELLIPTICITY OVER LARGE

FIELDS-OF-VIEW: THE FIRST STEP

TOWARDS ENABLING LSST WEAK LENSING SCIENCE

K. Schlaufman

November 5, 2004 
This document was prepared as an account of work sponsored by an agency of the United States Government. Neither the United States Government nor the University of California nor any of their employees, makes any warranty, express or implied, or assumes any legal liability or responsibility for the accuracy, completeness, or usefulness of any information, apparatus, product, or process disclosed, or represents that its use would not infringe privately owned rights. Reference herein to any specific commercial product, process, or service by trade name, trademark, manufacturer, or otherwise, does not necessarily constitute or imply its endorsement, recommendation, or favoring by the United States Government or the University of California. The views and opinions of authors expressed herein do not necessarily state or reflect those of the United States Government or the University of California, and shall not be used for advertising or product endorsement purposes.

This work was performed under the auspices of the U.S. Department of Energy by University of California, Lawrence Livermore National Laboratory under Contract W-7405-Eng-48. 


\title{
SIMULATIONS OF TURBULENCE INDUCED ELLIPTICITY OVER LARGE FIELDS-OF-VIEW: THE FIRST STEP TOWARDS ENABLING LSST WEAK LENSING SCIENCE
}

\author{
KEVIN SCHLAUFMAN
}

\begin{abstract}
Atmospheric turbulence can mimic the effects of weak lensing in astronomical images, so it is necessary to understand to what degree turbulence affects weak lensing measurements. In particular, we studied the ellipticity induced upon the point-spread functions (PSFs) of a grid of simulated stars separated by distances $\left(d \approx 1^{\prime}\right)$ that will be characteristic of Large Synoptic Survey Telescope (LSST) images. We observe that atmospherically induced ellipticity changes on small scales $\left(d<0.5^{\prime}\right)$ and use linear interpolation between stars separated by $d=0.5^{\prime}$ to determine the induced ellipticity everywhere in the field-of-view.
\end{abstract}

\section{The Code}

The optical effects of turbulence are usually restricted to the Earth's troposphere $(h<10 \mathrm{~km})$, with the greatest turbulence usually found near ground level, followed by an exponential decay. However, another peak exists at the tropopause due to the shearing effects of the jet stream. Still, in the end, the total difference in index of refraction over more than $10 \mathrm{~km}$ of atmosphere is only about 1 part in $10^{6}$.

The code that we used to simulate starlight's path through the Earth's turbulent atmosphere was developed in house at LLNL by a team led by Don Gavel and was initially used to simulate the performance of the Lick Observatory's adaptive optics systems. The code assumes that the atmospheric turbulence obeys a Kolmogorov Model over a range of spatial frequencies. The key point of the Kolmogorov Model of turbulence is that energy is added to the atmospheric fluid through large-scale perturbations (the so-called outer scale) which deteriorate into smaller scale disturbances. At the same time, kinetic energy is transferred from large-scale to small-scale structures, until the energy is eventually dissipated through viscous friction (the so-called inner scale). An analysis using these assumptions eventually leads to (see [1]) 


$$
\Phi(\kappa) \propto \kappa^{-5 / 3}
$$

Where $\Phi$ is spectral density of the turbulence and $\kappa=2 \pi / l$ is the spatial-wave number of a perturbation of size $l$. The low spatial frequency cutoff is specified by setting the parameter outerscale, while the high spatial frequency cutoff is determined by the sampling, set by the parameter sampInt; the inner scale is not specified by the user.

Taken together, these assumptions allow the code to treat the turbulence as both homogeneous and isotropic. The final assumption the code makes is the "frozen flow" hypothesis. In other words, it assumes that on the timescales of an LSST exposure $(\approx 10$ seconds) that the turbulence does not change - it is frozen in time and merely carried on the wind past the aperture. The code is therefore able to create static phase screens that describe the spatial distribution of the variation in index of rarefaction and simply translate them as the exposure is taken.

The necessary input parameters were also set to match the proposed LSST configuration:

(1) $\mathbf{D}=8.4 \mathrm{~m}$ - The diameter of the aperture

(2) expTime $=10.0 \mathrm{~s}$ - The total exposure time

(3) $\mathbf{d t}=0.2 \mathrm{~s}-$ The time between coadded images. That is, every dt seconds, the phase screens are translated by an amount equal to $v_{\text {wind }} d t$. The resulting images at each time step are then coadded together to produce the final result.

(4) $\mathbf{w l}=623 \times 10^{-9} \mathrm{~m}$ - The central wavelength of the filter

(5) $\mathbf{b w}=137 \times 10^{-9} \mathrm{~m}-$ The bandpass of the filter

Note that the values for $\mathbf{w l}$ and bw correspond to the Sloan $r^{\prime}$ filter.

\section{The Model Atmosphere}

The atmospheric model for the Cerro Pachon site implemented in our analysis can be found in [2]. It consists of seven discrete layers with total turbulence $r_{o, t o t}=0.2 \mathrm{~m}$. Each layer is described by 


$\begin{array}{ccccc}\begin{array}{c}\text { Layer } \\ \text { Number }\end{array} & \begin{array}{c}\text { Height } \\ (k m)\end{array} & \begin{array}{c}\text { Wind Speed } \\ \left(\mathrm{ms}^{-1}\right)\end{array} & \begin{array}{c}\% \\ \text { Atmosphere }\end{array} & \begin{array}{c}r_{o, i} \\ (\mathrm{~m})\end{array} \\ \mathbf{1} & 15.8 & 20.0 & 1.50 & 2.49 \\ \mathbf{2} & 13.1 & 10.0 & 8.00 & 0.910 \\ \mathbf{3} & 7.40 & 0.00 & 2.50 & 1.83 \\ \mathbf{4} & 5.80 & 0.00 & 3.50 & 1.49 \\ \mathbf{5} & 3.30 & 0.00 & 11.9 & 0.717 \\ \mathbf{6} & 1.80 & 0.00 & 7.80 & 0.924 \\ \mathbf{7} & 0.00 & 0.00 & 64.6 & 0.260\end{array}$

Where for every $i$ th layer

$$
r_{o, i}=r_{o, t o t}\left(\frac{\% \text { Atmosphere }}{100.0}\right)^{-3.0 / 5.0}
$$

\section{The Analysis}

We used the code to simulate the the effects of atmospheric turbulence on a regular grid of stars $5^{\prime}$ on a side with separation $d=.5^{\prime}-$ a total of 441 artificial point sources. See Figure 1 for a typical PSF.

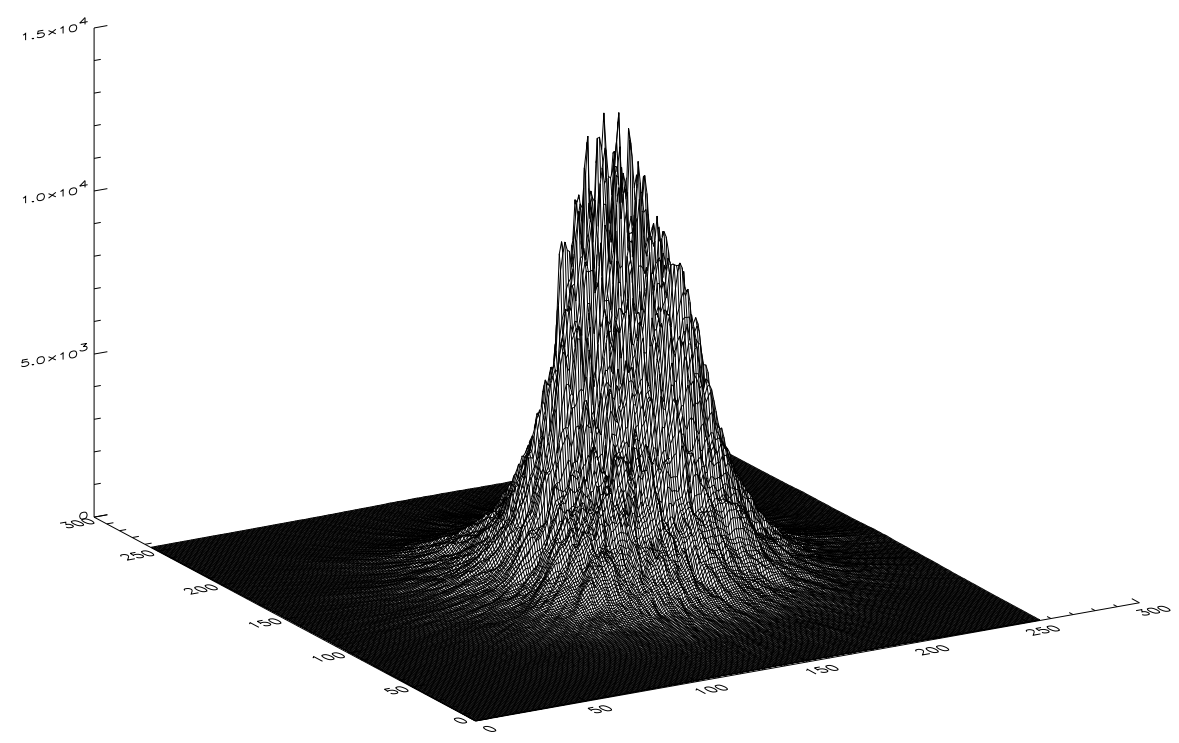

We used the IDL routine gauss2dfit to find the centroid of each stellar image. With the centroid $\left(x_{c}, y_{c}\right)$ in hand, let $I(x, y)$ be the intensity as a function of $x$ and $y$; then the ellipticity is computed: 


$$
\begin{gathered}
<x^{2}>=\frac{\sum\left(I(x, y)\left|x-x_{c}\right|^{2}\right)}{\sum I(x, y)} \\
<y^{2}>=\frac{\sum\left(I(x, y)\left|y-y_{c}\right|^{2}\right)}{\sum I(x, y)} \\
<x y>=\frac{\sum\left(I(x, y)\left(x-x_{c}\right)\left(y-y_{c}\right)\right)}{\sum I(x, y)}
\end{gathered}
$$

Finally

$$
\begin{gathered}
E_{1}=\frac{<x^{2}>-<y^{2}>}{<x^{2}>+<y^{2}>} \\
E_{2}=\frac{2<x y>}{<x^{2}>+<y^{2}>} \\
E=\left(E_{1}^{2}+E_{2}^{2}\right)^{1 / 2}
\end{gathered}
$$

We therefore had a grid of 441 nodes of known ellipticity. Using this information and the IDL bilinear routine, we were able to use bilinear interpolation to compute the ellipticity anywhere in the field-of-view as function of $x$ and $y$. Contour plots of $E(x, y)$, as well as $E_{1}(x, y)$ and $E_{2}(x, y)$ are included in Figures 2, 3, and 4; note that the contours are 0.1 units apart in each case and that each plot is $5^{\prime}=300^{\prime \prime}$ on a side.

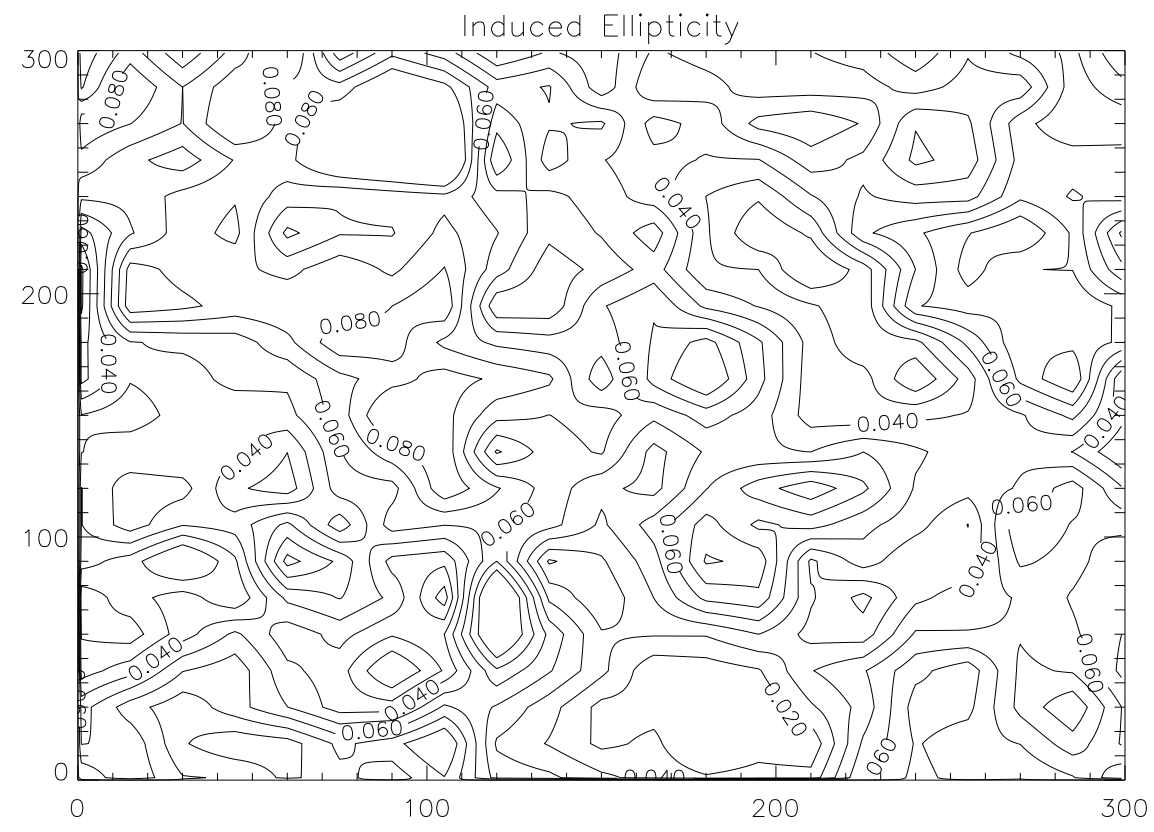





To test the accuracy of the bilinear interpolation, we removed one at a time each node where bilinear interpolation was possible (everywhere but on the edges of the grid) and then used bilinear to compute an 
estimate for the ellipticity at that location based upon the remaining nodes. The absolute error in interpolation could then be calculated at every interior node. The histogram in Figure 5 shoes the results of binning these absolute errors in 0.002 unit wide bins.

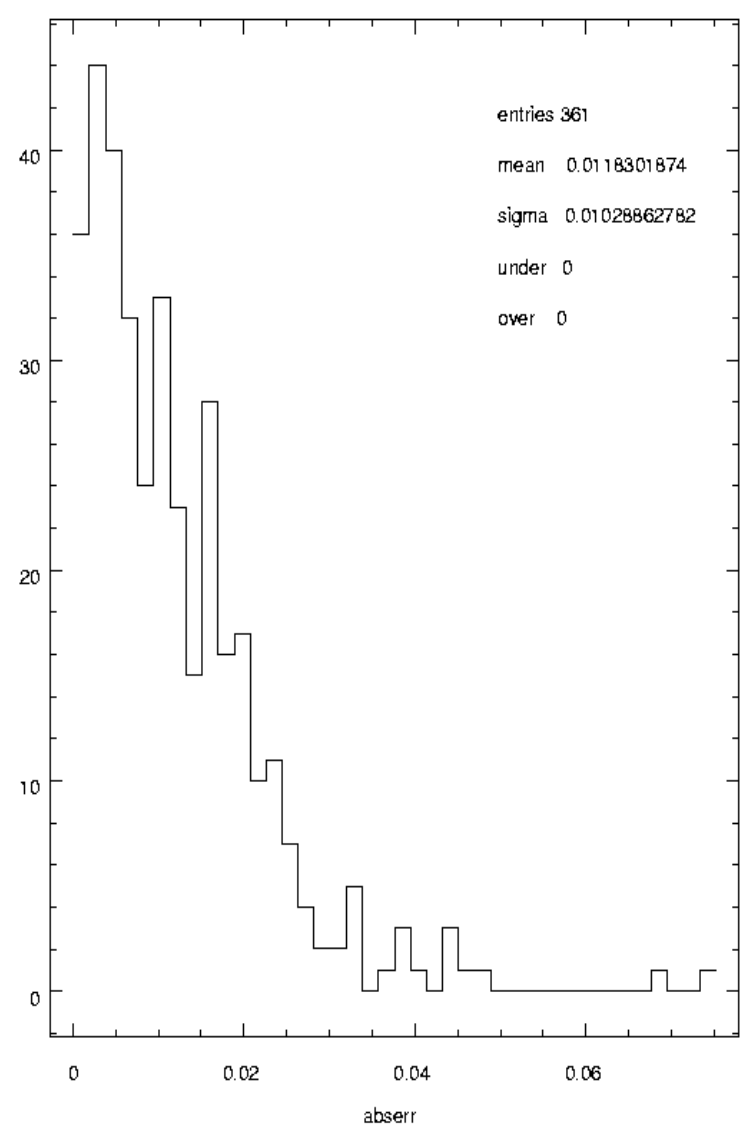

Note how there are only a few nodes where the interpolation returned a significant absolute error. This fact implies that linear interpolation between nodes separated by $d \approx 1^{\prime}$ may be accurate enough to remove the effects of atmospheric turbulence from future LSST weak lensing observations.

\section{The Conclusions}

These results are promising, and it seems that weak lensing science on massive scale will become a reality with the LSST. Still, there a few caveats to keep in mind: 
(1) An incomplete atmospheric model was used in this analysis, as there was no wind information included for most of the layers.

(2) Correlations may exist across longer scales in the induced ellipticities. Investigating much larger fields-of-view is not computationally practical right now, however.

Still, this analysis indicates that linear interpolation does not introduce unacceptably large errors when it is used to infer the atmospherically induced ellipticity at arbitrary points in the field-of-view from a regularly sampled grid spread evenly across that field-of-view.

\section{REFERENCES}

[1] Hardy, J. W. 1998, Adaptive Optics for Astronomical Telescopes, Oxford University Press.

[2] Ellerbroek, B. L. \& Rigaut, F. J. 2000, PSPIE, 4007, 1088 